\title{
THE FINE STRUCTURE OF
}

\section{MOTOR ENDPLATE MORPHOGENESIS}

\author{
A. M. KELLY and S. I. ZACKS \\ From the Department of Pathology, School of Veterinary Medicine, University of Pennsylvania, \\ and The Ayer Clinical Laboratory, Pennsylvania Hospital, Philadelphia, Pennsylvania 19104
}

\begin{abstract}
A BSTRACT
The fine structure of the developing neuromuscular junction of rat intercostal muscle has been studied from 16 days in utero to 10 days postpartum. At 16 days, neuromuscular relations consist of close membrane apposition between clusters of axons and groups of myotubes. Focal electron-opaque membrane specializations more intimately connect axon and myotube membranes to each other. What relation these focal contacts bear to future motor endplates is undetermined. The presence of a group of axons lying within a depression in a myotube wall and local thickening of myotube membranes with some overlying basal lamina indicates primitive motor endplate differentiation. At 18 days, large myotubes surrounded by new generations of small muscle cells occur in groups. Clusters of terminal axon sprouts mutually innervate large myotubes and adjacent small muscle cells within the groups. Nerve is separated from muscle plasma membranes by synaptic gaps partially filled by basal lamina. The plasma membranes of large myotubes, where innervated, simulate postsynaptic membranes. At birth, intercostal muscle is composed of separate myofibers. Soleplate nuclei arise coincident with the peripheral migration of myofiber nuclei. A possible source of soleplate nuclei from lateral fusion of small cells' neighboring areas of innervation is suspected but not proven. Adjacent large and small myofibers are mutually innervated by terminal axon networks contained within single Schwann cells. Primary and secondary synaptic clefts are rudimentary. By 10 days, some differentiating motor endplates simulate endplates of mature muscle. Processes of Schwann cells cover primary synaptic clefts. Axon sprouts lie within the primary clefts and are separated from each other. Specific neural control over individual myofibers may occur after neural processes are segregated in this manner.
\end{abstract}

\section{INTRODUCTION}

Numerous attempts have been made to unravel the architectural complexities of motor endplates by studying their morphogenesis. This approach has been fraught with difficulty for, apart from the limited resolving power of the light microscope, available staining techniques did not permit visualization, in the same tissue section, of the distribution of terminal axons and the configuration of the muscle surfaces innervated by these axons. Consequently, considerable confusion has arisen concerning time of initial neuromuscular contact and the commencement of motor endplate differentiation. Many classic studies suggest that nerve endings are related to primitive muscle cells early in myogenesis $(7,9)$. For example, in the forelimb of the rat, morphological studies by East (1931) and by Strauss and Weddell (1940) indicate neuromuscular contact at 16 days' gestation. This stage corresponds with the stage at which Windle et al. (1935) and Strauss and 
Weddell (1940) were able to elicit muscular contractions by neural stimulation. However, these authors found no evidence of motor endplate differentiation at these stages. In fetal mice, Couteaux (1960) interpreted early neuromuscular contacts as nonspecific since there was no discernible endplate formation. He suggested that motor endplate differentiation does not commence until after muscle cells have matured to myofibers. Histochemical studies of the development of acetylcholinesterase activity by Kupfer and Koelle (1951) support this interpretation. In contrast, more recent histochemical studies in several species including the rat indicate that motor endplates form on muscle cells before peripheral migration of muscle cell nuclei $(11,13,20,28)$. Morphological studies of motor endplate formation in human fetuses by Cuajunco (1942) also suggest that endplate formation occurs on myotubes.

Many of these differences may be resolved by a study of the development of neuromuscular relationships with the electron microscope. This approach has been used by Bleckschmidt and Daikoku (1966) and by Hirano (1967), but these authors only describe events of neuromuscular development after muscle cells have matured to myofibers. Kelly and Zacks (1966) described evidence of motor endplate differentiation on the intercostal myotubes of 18-day rat fetuses, and their findings have been confirmed recently by Teravainen (1968).

The present study describes early neuromuscular relations and the development of motor endplates in rat intercostal muscle and correlates these development events with the pattern of histogenesis of this muscle. Intercostal muscle was chosen because its centrally located innervation band facilitated selection of areas of neuromuscular contact.

\section{MATERIALS AND METHODS}

Skeletal muscle was obtained from the proximal third of the rib cage from individual rats of two separate litters of Sprague-Dawley rats at each of the following intervals: 16, 18, and 20 days in utero, birth (after 22 days' gestation), and 10 days postpartum. No one fixative proved satisfactory for the series of developmental stages, and best results were obtained by use of the following:

16-day fetus: $2.5 \%$ glutaraldehyde, $4 \%$ paraformaldehyde in phosphate buffer (pH 7.2) (Trelstad et al., 1967); 18-day fetus: $6 \%$ glutaraldehyde in phosphate

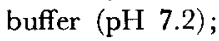

20-day fetus; $2.5 \%$ glutaraldehyde, $1 \%$ paraformaldehyde in phosphate buffer ( $\mathrm{pH} \mathrm{7.2);}$

Birth and 10 days postpartum: $5 \%$ glutaraldehyde, $4 \%$ paraformaldehyde in phosphate buffer $(\mathrm{pH} 7.2)$ (Karnovsky, 1965).

After fixation, tissues were postfixed with Dalton's solution (8), flat-embedded in Araldite (Ciba), orientated, cut into small blocks and affixed to plastic rods. $0.2-1 \mu$ sections cut from these blocks were mounted on glass slides and stained with a heated solution of $0.1 \%$ crystal violet in $1 \%$ sodium borate.

At each of the stages examined, material was transversely orientated and sectioned serially so as to obtain as complete a picture as possible of the interaction between nerve and muscle. Thin sections were stained with uranyl acetate and lead citrate and examined in an RCA EMU III F electron microscope. Acetylcholinesterase activity was demonstrated by the thiolacetic acid method (1) in unfixed tissue. Whole mounts of unfixed rib cages stained for acetylcholinesterase were examined in the light microscope.

\section{OBSERVATIONS}

The following description presents morphological features which appear to be most representative of developing motor endplates at 16, 18, and 20 days in utero, birth, and 10 days postpartum.

At 16 days in utero, myotubes were frequently clustered into groups within which the plasma membranes of neighboring muscle cells were interconnected by close and tight junctions (17). Morphologically undifferentiated cells surrounded these groups of myotubes and were interconnected with the myotube membranes by close junctions. No basal lamina surrounded these groups of muscle cells (17).

Intramuscular nerves coursed through the tissue between the groups of muscle cells (Fig. 1). They were composed of loose aggregates of juxtaposed axons loosely and incompletely enwrapped by immature Schwann cells. Morphological criteria for distinguishing axon processes from Schwann cell processes were based on previous studies by Peters and Muir (1959) and Hirano (1967). Axon processes were identified by their electron-lucent cytoplasm containing neurotubules, but no ribosomes. As axons approached muscle cells, neurotubules were less frequent and vesicles appeared within the cytoplasm (Figs. 2 and 3). The largest vesicles, 700-900 $\mathrm{A}$ in diameter, had electron-opaque cores and appeared 




FigUne 116 days' gestation. An intercostal nerve neighbors a group of myotubes. The nerve is composed of a loose aggregate of juxtaposed axons and is incompletely enwrapped by immature Schwann cells. Axon cytoplasm is electron lucent and is distinct from the ribosome-studded cytoplasm of the primitive Schwann cells. Two axons (arrowed) lie between the Schwann cell and the myotube surface membranes. $\times 5,000$. Marker $=1 \mu$.

similar to vesicles associated with catecholamine storage in adrenergic nerves (21). Similar large vesicles at developing endplates have previously been described by Teravainen (1968). Smaller vesicles, $500 \mathrm{~A}$ in diameter, with electron-lucent centers, which are more typical of synaptic vesicles in cholinergic axons, were intermingled with the large cored vesicles. As there was no particular concentration of these vesicles in areas of neuromuscular contact comparable to the concentration found in more differentiated endplates, it was not possible to distinguish terminal from nonterminal axons. Characteristically, axons of comparable size were clustered together in groups which were peripherally enwrapped by Schwann cell processes (Fig. 2). Cytoplasmic content of ribosomes, profiles of rough endoplasmic reticulum, and proximity to axons identified Schwann cells $(12,22)$. There was no myelin at this time.

Plasma membranes of axons and accompanying Schwann cells came into close association with muscle cell membranes (Fig. 2). In some of these associations, the intercellular space measured less than $100 \mathrm{~A}$ and there were focal electron-opaque 
specializations in the membranes resembling close junctions (Fig. 3). Similar close membrane associations also occurred between axons and morphologically undifferentiated cells (Fig. 4). These neuromuscular contacts occurred in the mid-parts of developing intercostal muscles where future motor endplates were expected to form. Some neuromuscular contacts may have been associated with the development of muscle spindles since it was not possible to distinguish intrafusal from extrafusal fibers at 16 days. However, as groups of axons traced in serial sections were observed to come into close association with several neighboring groups of myotubes, it seems likely that extrafusal fibers were included amongst them. Close membrane association also occurred between axon trunks and myotube membranes in areas where the layer of Schwann cells was incomplete (Fig. 5).

Myotubes at this stage had no morphological features to suggest why a particular area of their plasma membranes was selected for neural contact; there were no aggregates of sarcoplasm or myotube nuclei in areas of neuromuscular apposition (Fig. 2). In addition to the close junctions present between nerve and muscle at 16 days, rare neuromuscular contacts more suggestive of future motor endplate development were observed. Although difficult to preserve adequately, these areas consisted of a cluster of axon sprouts lying in a shallow depression in the myotube wall, but separated from the myotube plasma membrane by a gap partially filled with amorphous material suggesting a rudimentary basal lamina (Fig. 6). In addition, the muscle surface membrane had focal thickenings resembling those described by Lentz (1968) in Triturus.

At 18 days, the developing muscle was composed of myotubes measuring up to $20 \mu^{2}$ with undifferentiated cells and new generations of small muscle cells clustered around their walls. Each of these cell complexes was ensheathed by a basal

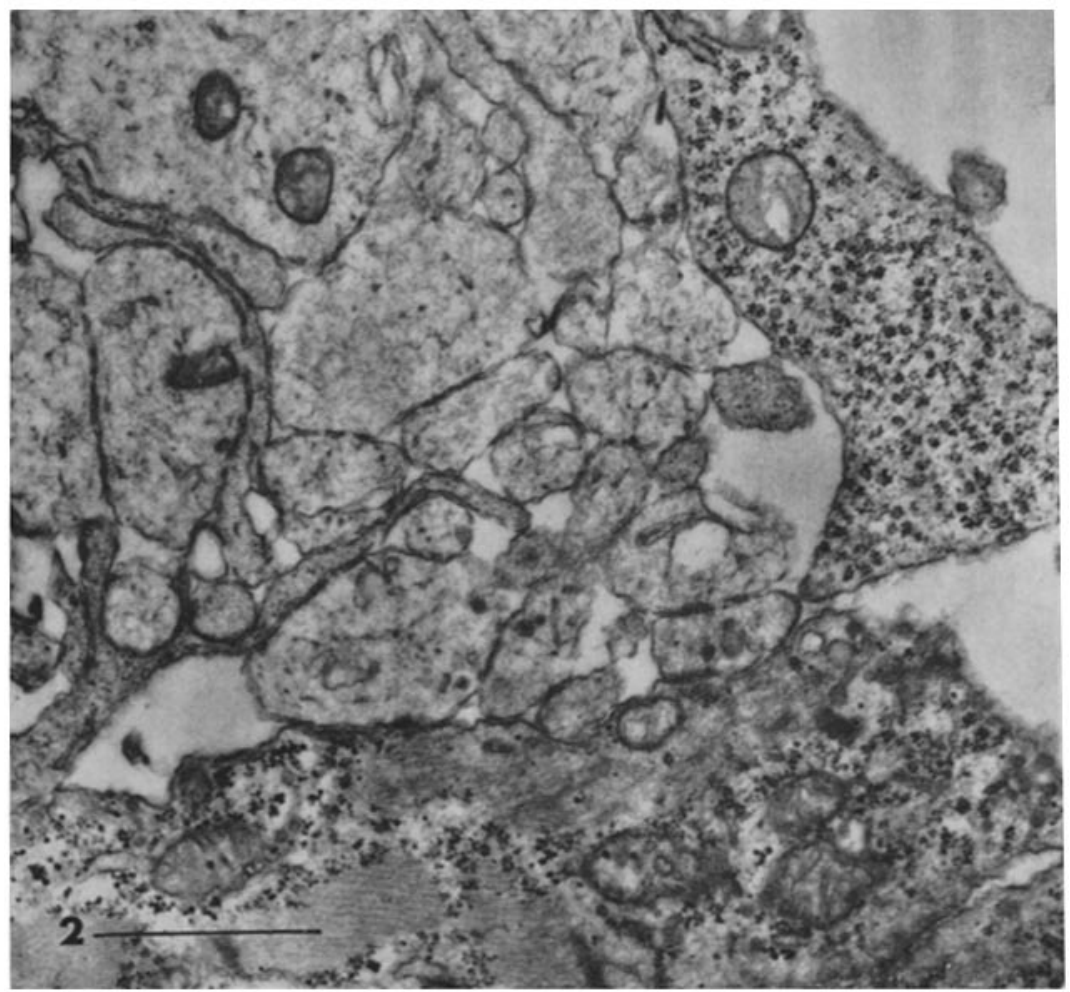

Figure 216 days' gestation. The plasma membranes of axons and accompanying Schwann cells are in close association with muscle cell membranes. Neurotubules within axon processes are diminished in these areas of neuromuscular contact. $\times 23,500$. Marker $=1 \mu$. 


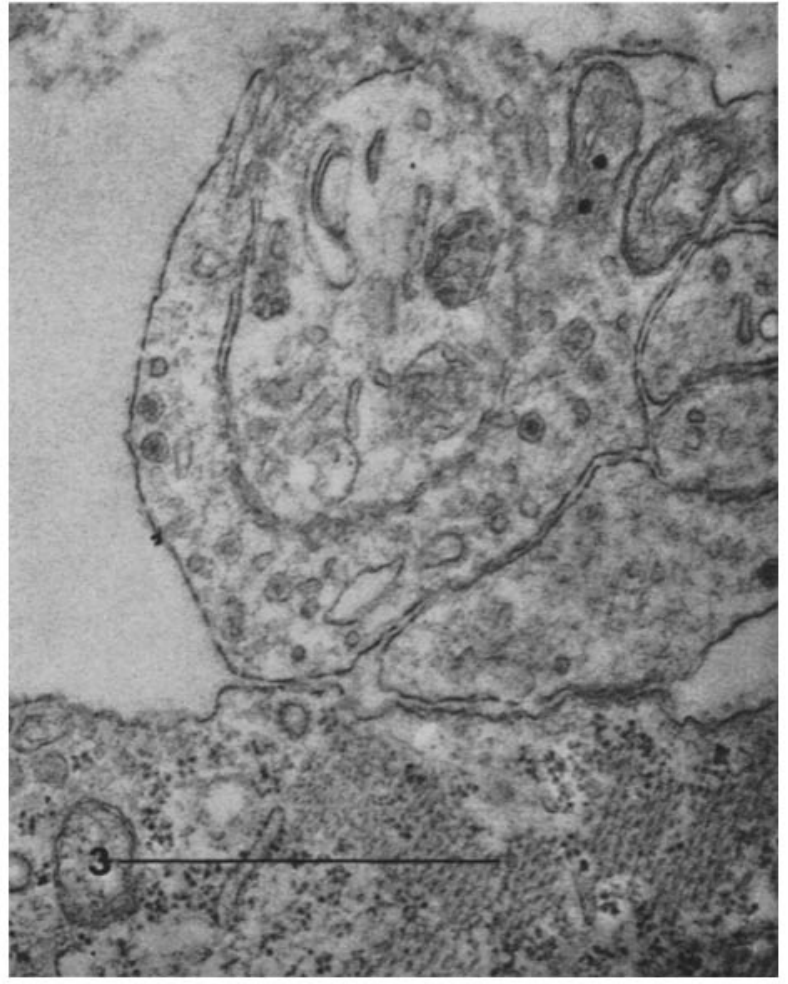

Figure 316 days' gestation. Plasma membranes of two-axon sprouts are in close association with those of a muscle cell. An electron-opaque contact more intimately relates these apposed membranes. Vesicles with electron-opaque cores occur within the axon processes. $\times 38,000$. Marker $=$ $1 \mu$.

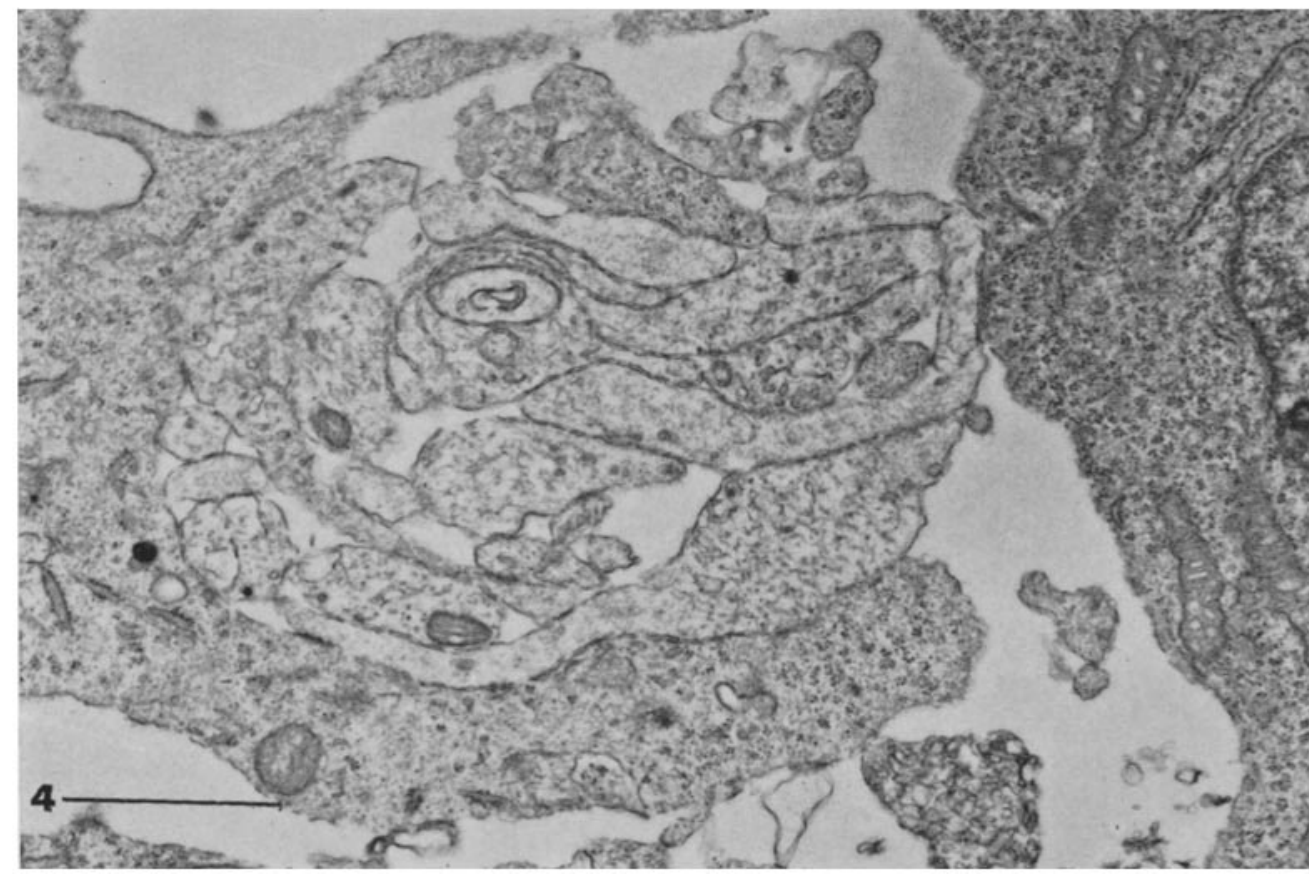

FIGURE 416 days' gestation. A group of axon sprouts which are loosely ensheathed by a Schwann cell lie in close association with the plasma membranes of a morphologically undifferentiated cell. $\times 22,600$. Marker $=1 \mu$. 


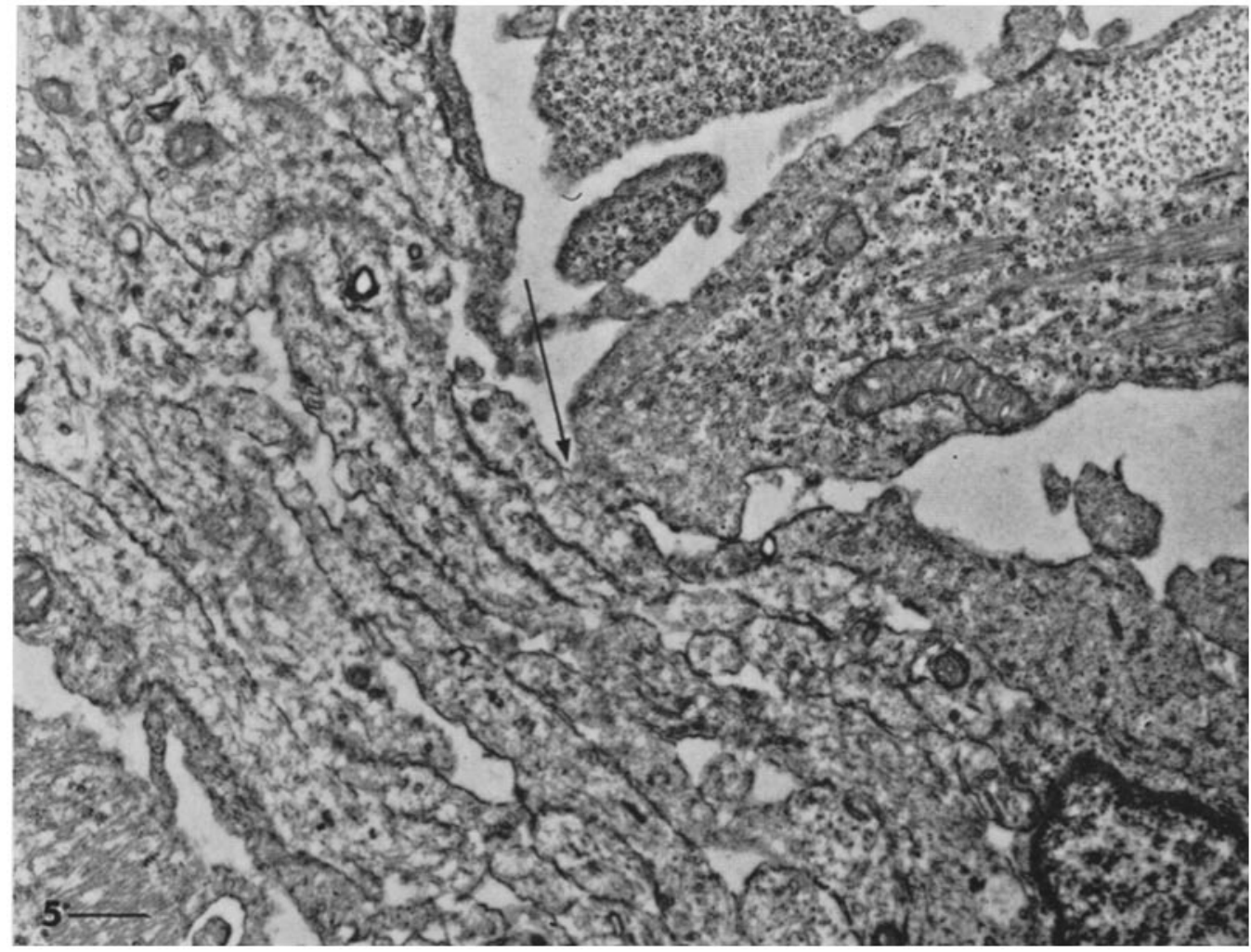

Figure 516 days' gestation. Longitudinal section of an intercostal nerve which is incompletely enwrapped by Schwann cells. The membranes of a myotube appear to come into contact with an axon trunk (arrowed). $\times 9,000$. Marker $=1 \mu$.

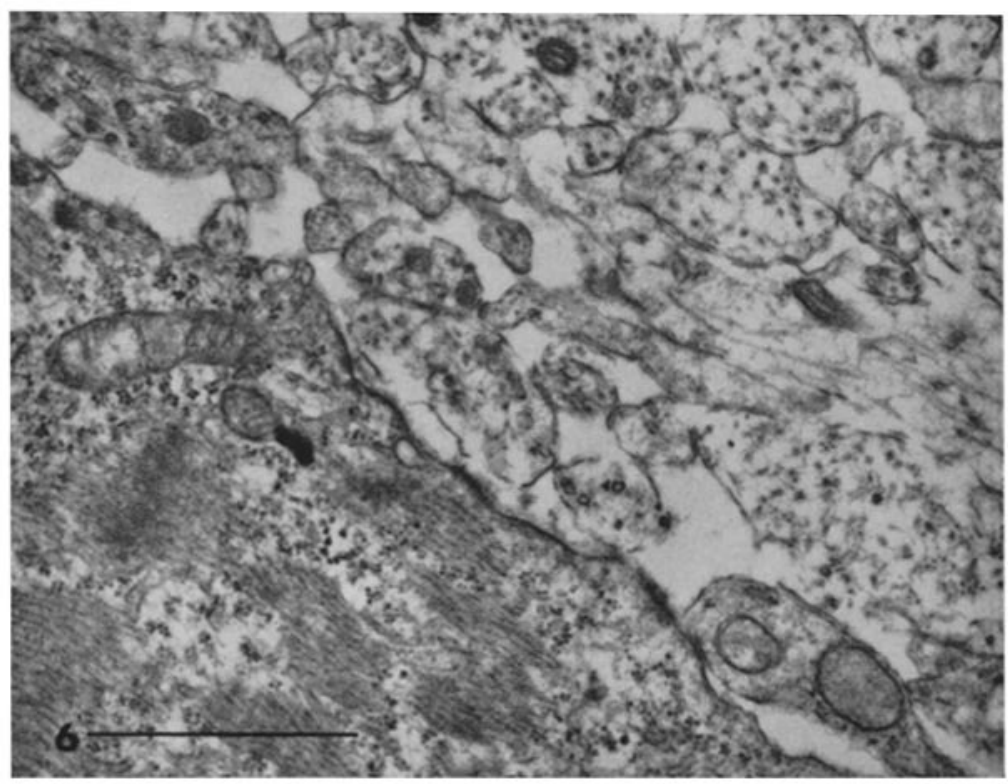

Figure 616 days' gestation. A cluster of axon sprouts occupy a shallow depression of the wall of a myotube. The axon sprouts are separated from the myotube plasma membranes by a gap which is partially filled by amorphous material which is interpreted as rudimentary basal lamina. $\times 27,000$. Marker $=1 \mu$. 


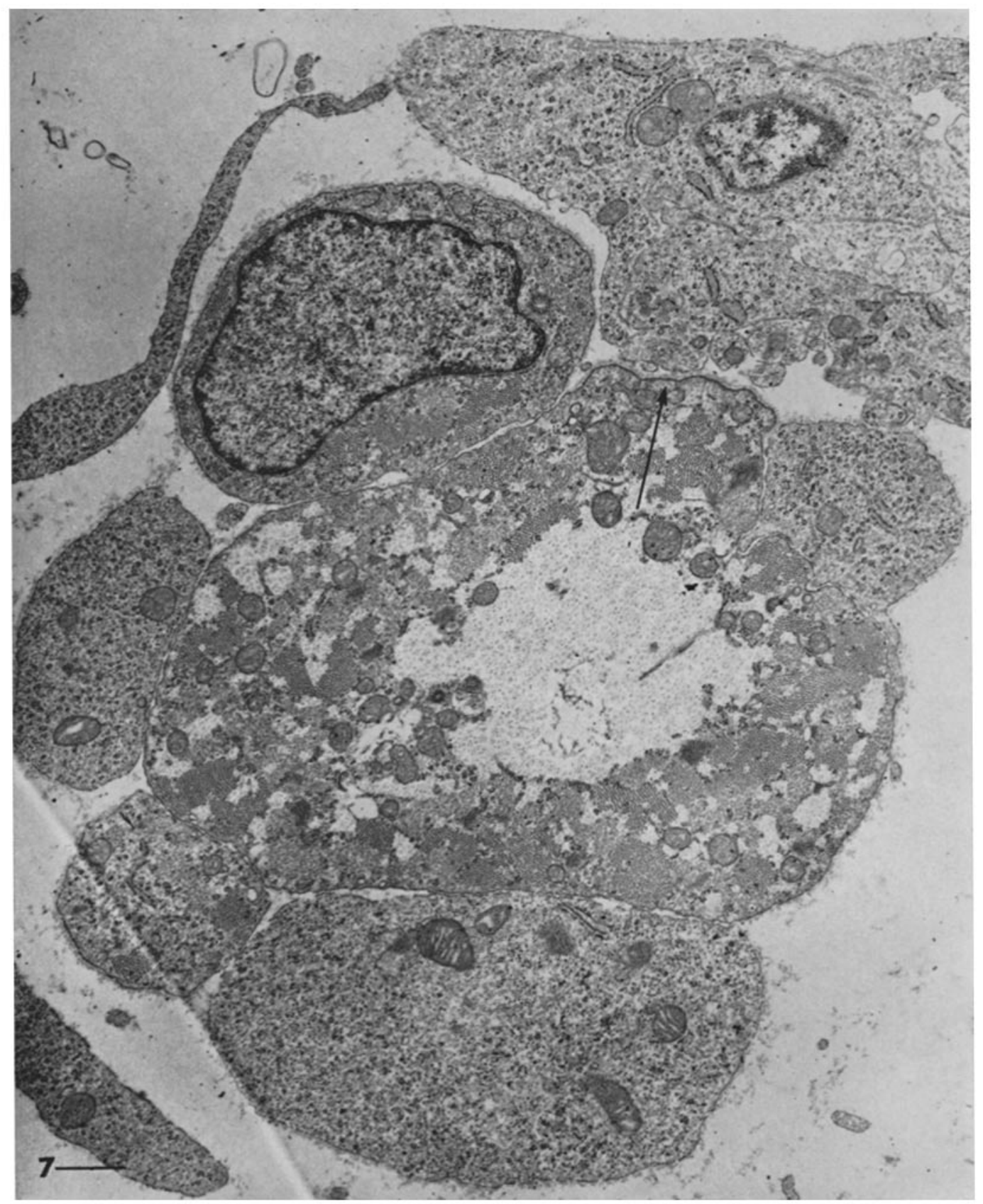

FIGURE 718 days' gestation. A large myotube which contains much glycogen is surrounded by new generations of small cells. Two of the new small cells contain myofibrils. A group of axon sprouts approach the membranes of the large myotube. At this area of contact, the myotube membranes are locally thickened and electron opaque (arrowed). $\times 10,000$. Marker $=1 \mu$. 


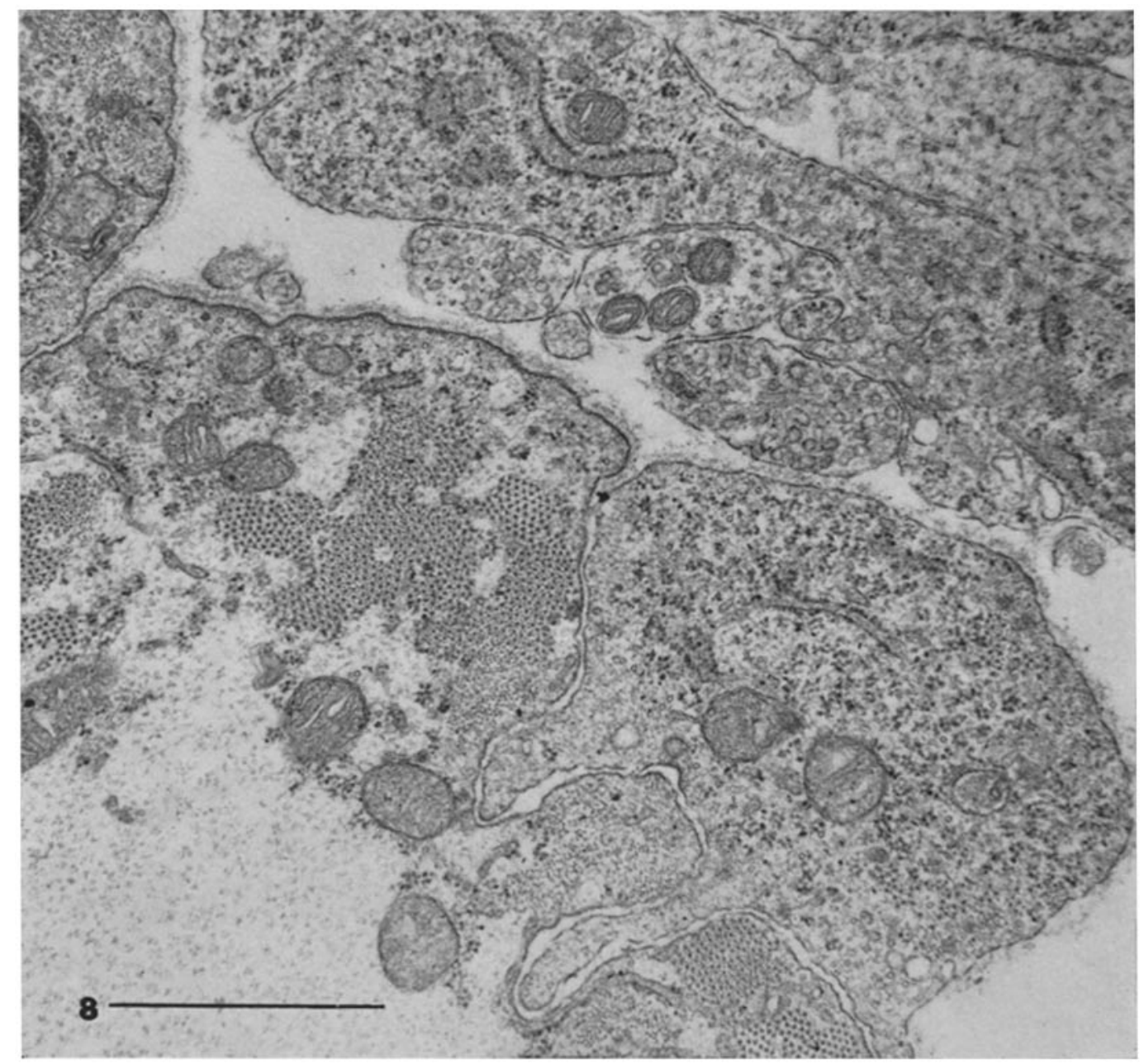

Figure 818 days' gestation. Higher power of area of neuromuscular contact shown in Fig. 7. A small undifferentiated cell lies in intimate and complex relationship with the large myotube. Both cells are mutually innervated by groups of terminal axon sprouts which contain numerous synaptic vesicles. Axon sprouts are separated from muscle membranes by basal lamina. The myotube plasma membrane and basal lamina are locally thickened and electron opaque, suggesting the early differentiation of a postsynaptic membrane. There is no such specialization of the membranes of the undifferentiated cell. $\times 33,500$. Marker $=1 \mu$.

lamina which did not penetrate between apposed cell membranes within the groups (17).

Peripheral Schwann cell sheaths more completely surrounded the primitive intramuscular nerves than at 16 days, but few Schwann cell processes penetrated between axons in the central portion of the nerve bundle. There was no myelin. At areas of innervation, axon sprouts contained concentrations of vesicles which were approximately $500 \mathrm{~A}$ in diameter and had electron-lucent centers (Figs. 7 and 8). These axon sprouts re- sembled the terminal axons of mature motor endplates. Some large vesicles with electron-opaque cores, "coated vesicles" (Roth and Porter, 1964), and neurotubules were also present within axon sprouts at areas of innervation.

Groups of three to six axon sprouts contained within a single Schwann cell approached clusters of extrafusal muscle cells, but there was no close association between nerve and muscle membranes as at 16 days. In every instance, axon sprouts were separated from myotube plasma membranes 


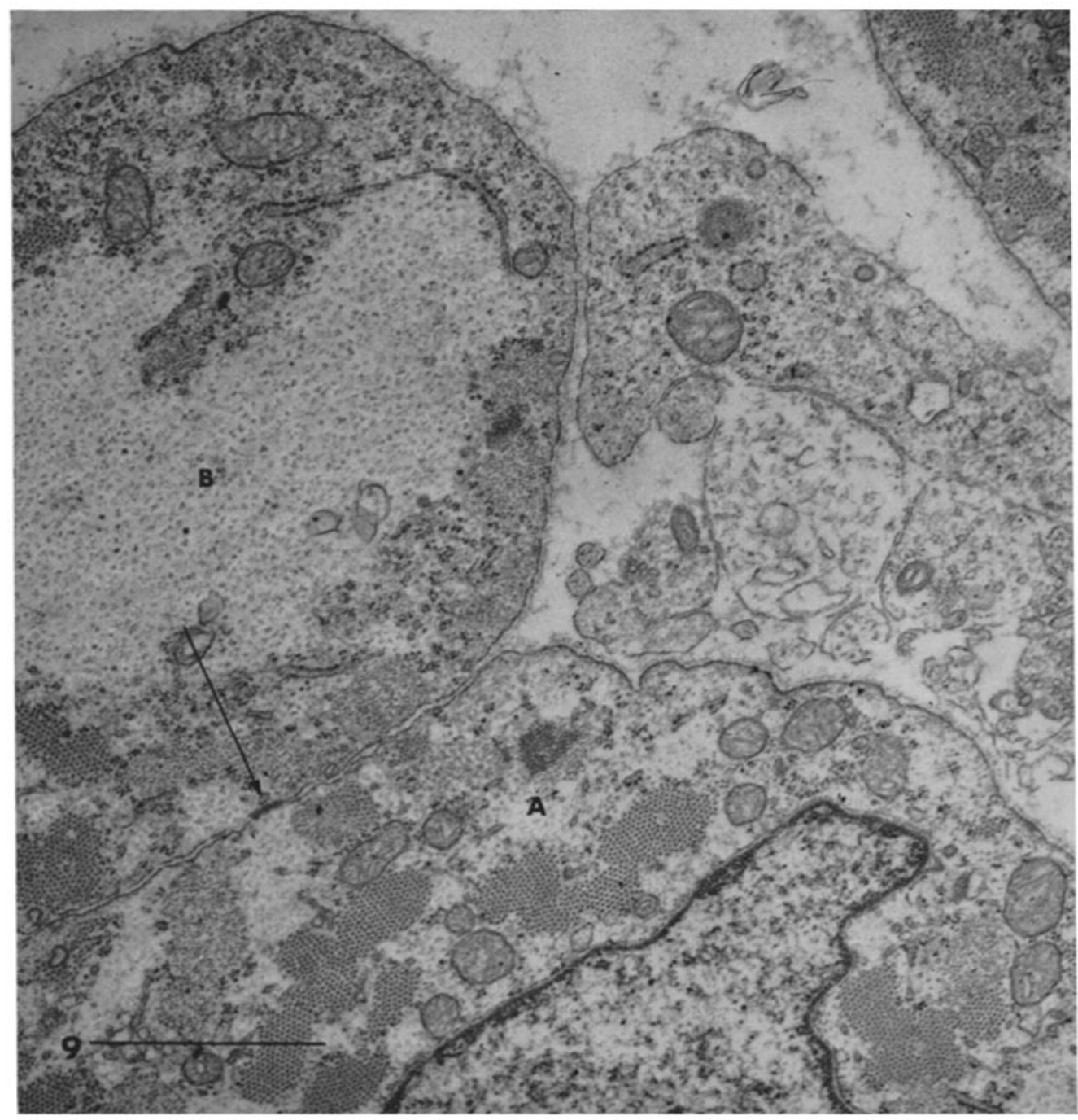

FIGURE 918 days' gestation. A group of axon sprouts innervate a large myotube $(A)$. Locally the myotube membranes are increased in electron opacity, and small invaginations suggest early development of synaptic clefts. A small myotube $(B)$ lies adjacent to the large myotube, and an electron-opaque membrane junction interconnects the membranes of the two cells (arrowed). $\times 29,500$. Marker $=1 \mu$.

by a synaptic gap measuring $500-900 \mathrm{~A}$ which was partially filled by basal lamina. The basal lamina ensheathing each aggregate of muscle cells reached its greatest breadth and density in gaps separating axon and myotube membranes (Fig. 8). The plasma membranes of myotubes underlying these areas of early innervation were wide and had increased electron opacity. This is the earliest definitive indication of the development of a typical postsynaptic membrane. Super- ficial basal lamina-lined depressions and furrows in these primitive postsynaptic membranes were interpreted as probable rudimentary primary and secondary synaptic clefts (Fig. 9).

Undifferentiated cells and new generations of small muscle cells frequently lay along the walls of large myotubes on either side of developing postsynaptic membranes (Fig. 7). As previously described (17), these new generations of small muscle cells are interconnected with the large 


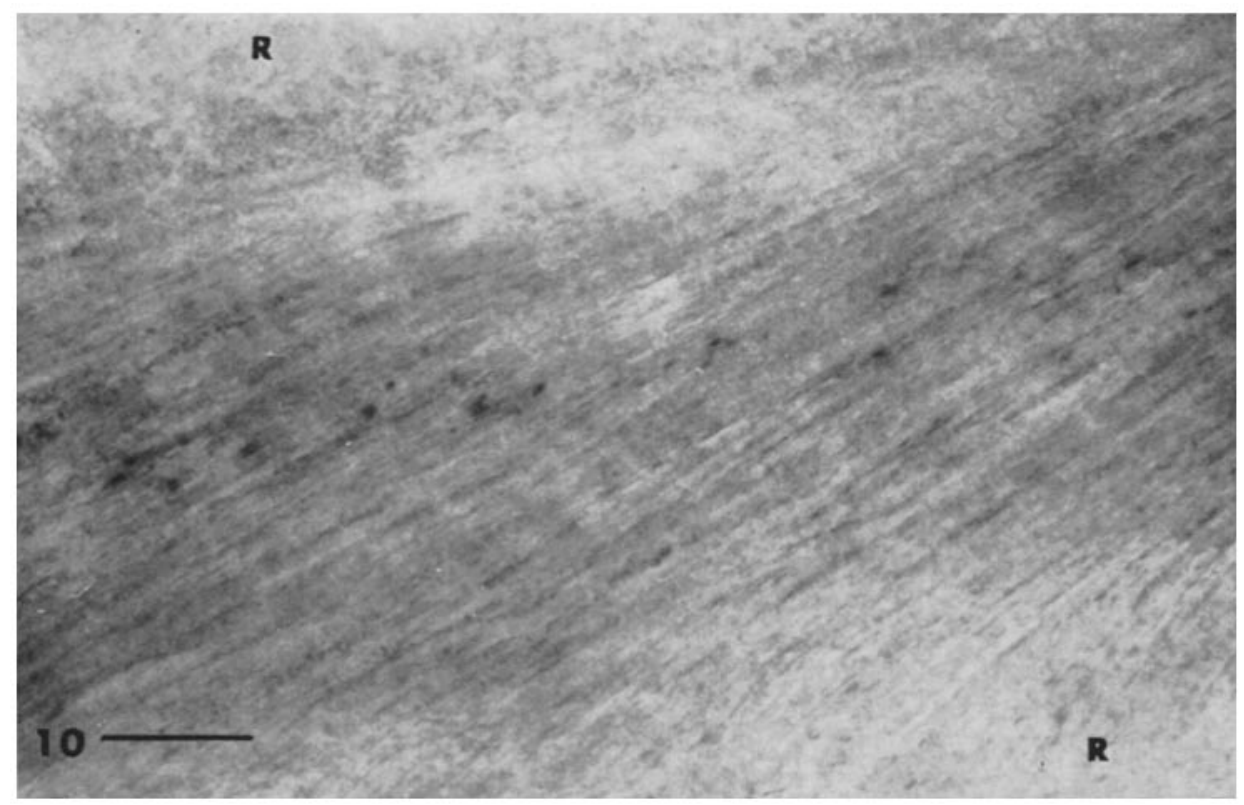

FiguRe 1018 days' gestation. Light micrograph of intercostal muscle stained by the thiolacetic acid method. Acetylcholinesterase activity at primitive endplates is indicated by focal deposits of lead which lie in a band in the center of the muscle, midway between two ribs $(R) . \times 150$. Marker $=100 \mu$.

myotubes by membrane specializations including close and tight junctions (see also Fig. 9). Frequently, a small cell was found which occupied a plasmalemmal depression adjacent to the developing postsynaptic membrane of a large myotube. Pseudopodia from such small cells entered tubular invaginations in the walls of the large myotubes (Fig. 8). The outlines of these closely related cells merged and axon sprouts followed their peripheral contour, innervating both the large myotube and the adjacent small cells. When traced in serial sections, the neural contacts with small muscle cells at 18 days had no focal thickening of the muscle cell membrane.

The thiolacetic acid method demonstrated foci of acetylcholinesterase activity in the centers of intercostal muscle from 18-day fetal rats, in sites where endplate formation was anticipated (Fig. 10). However, no organized subneural apparatus could be discerned by light microscopy at this time.

At 20 days in utero, intercostal muscle organization and endplate morphology were comparable to those at 18 days. Schwann cells surrounding intramuscular nerves frequently insinuated processes between groups of axons and thereby iso- lated one group from another. There was no myelin. Depressions in the postsynaptic membranes of large myotubes, suggestive of formative primary synaptic clefts, were more clearly defined than before. Small accumulations of sarcoplasm lay beneath postsynaptic membranes, and occasional nuclei were displaced from the myotube centers towards the developing endplate as if migrating in that direction. These changes indicated the early development of the sole plate. Small cells containing myofilaments lay in similar relation to the primitive motor endplates as at 18 days.

At birth, the developing muscle was composed of separate myofibers containing peripheral nuclei. The myofibers varied in size and intermingled in a checkerboard pattern (17). Axons of intramuscular nerve trunks were separated from each other by Schwann cell processes and there was early myelin formation. Innervating networks of terminal axon sprouts, in intimate contact with each other, made synaptic contact with the walls of large and neighboring small myofibers (Figs. 1 I and 12). Numerous synaptic vesicles of the cholinergic type and small osmiophilic granules measuring $150-300 \mathrm{~A}$ and resembling glycogen 


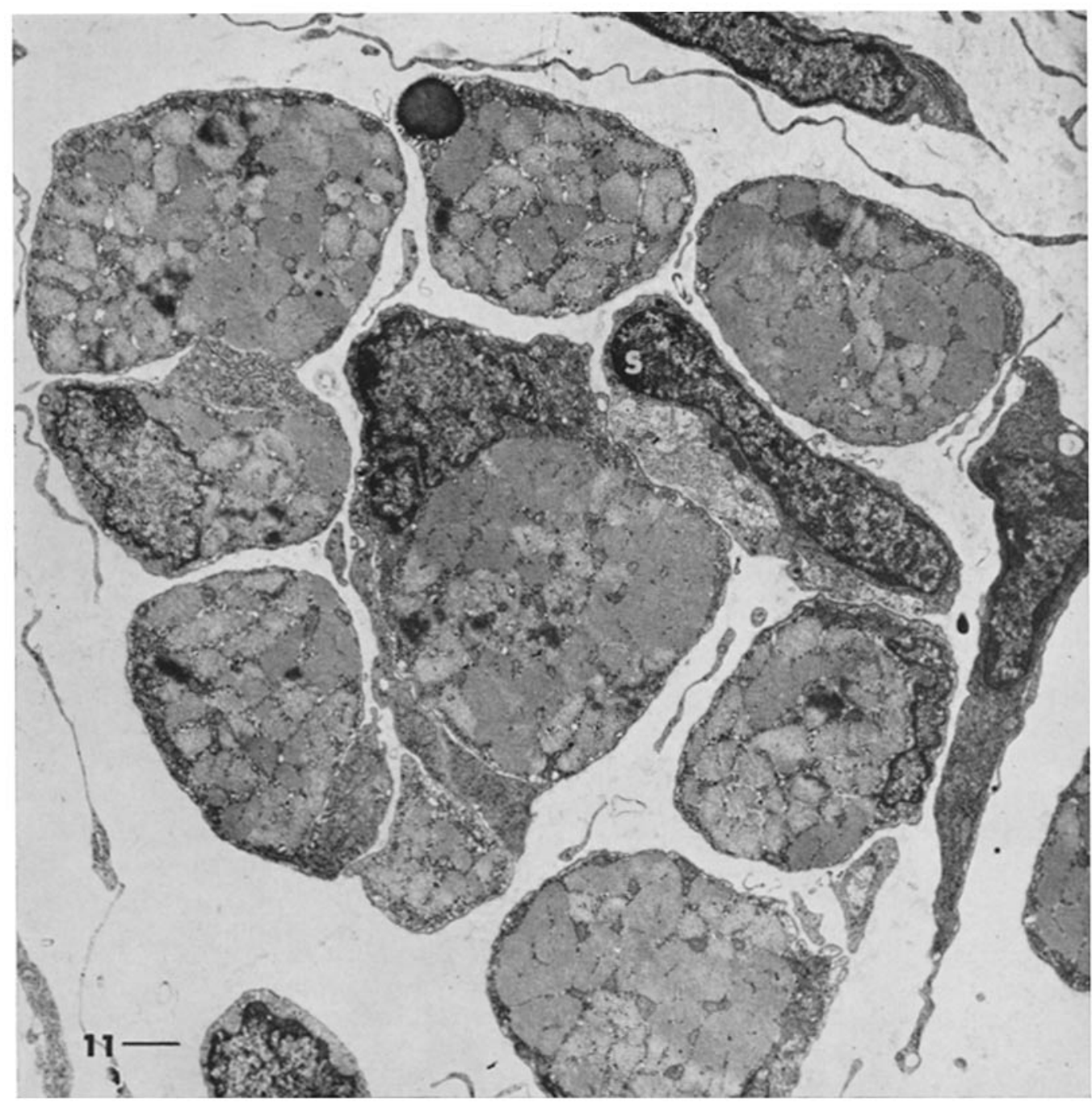

Figure 11 Birth. Large and small myofibers with peripheral nuclei lie independent of each other. A Schwann cell $(S)$, partially surrounding a network of axon sprouts, spans the gap between two neighboring muscle cells. A nucleus and some sarcoplasm lie to one side of the area of innervation on the large myofiber. $\times 7,000$. Marker $=1 \mu$.

were present within terminal nerve branches of all sizes, but were most common in the centers and towards the nonsynaptic surface of the large nerve sprouts. Dense-core vesicles of the adrenergic type and coated vesicles were infrequent.

Postsynaptic membranes of large myofibers partially surrounded some axon branches and formed shallow primary synaptic clefts (Fig. 13). Short and wide secondary synaptic clefts arose irregularly from primary clefts and also occurred within the entire postsynaptic membrane without relation to immature primary clefts. Frequently, small "pinocytic-like," noncoated vesicles appeared to bud from the tips of the secondary clefts. One or two soleplate nuclei and some sarcoplasm were aggregated either beneath or, more commonly, to one side of the postsynaptic membranes of the large myofibers. Thus, the peripheral location of myofibe: nuclei and the appearance of soleplate nuclei coincided. We obtained no evidence of any particular "endplate attraction" for nuclei. For example, several 


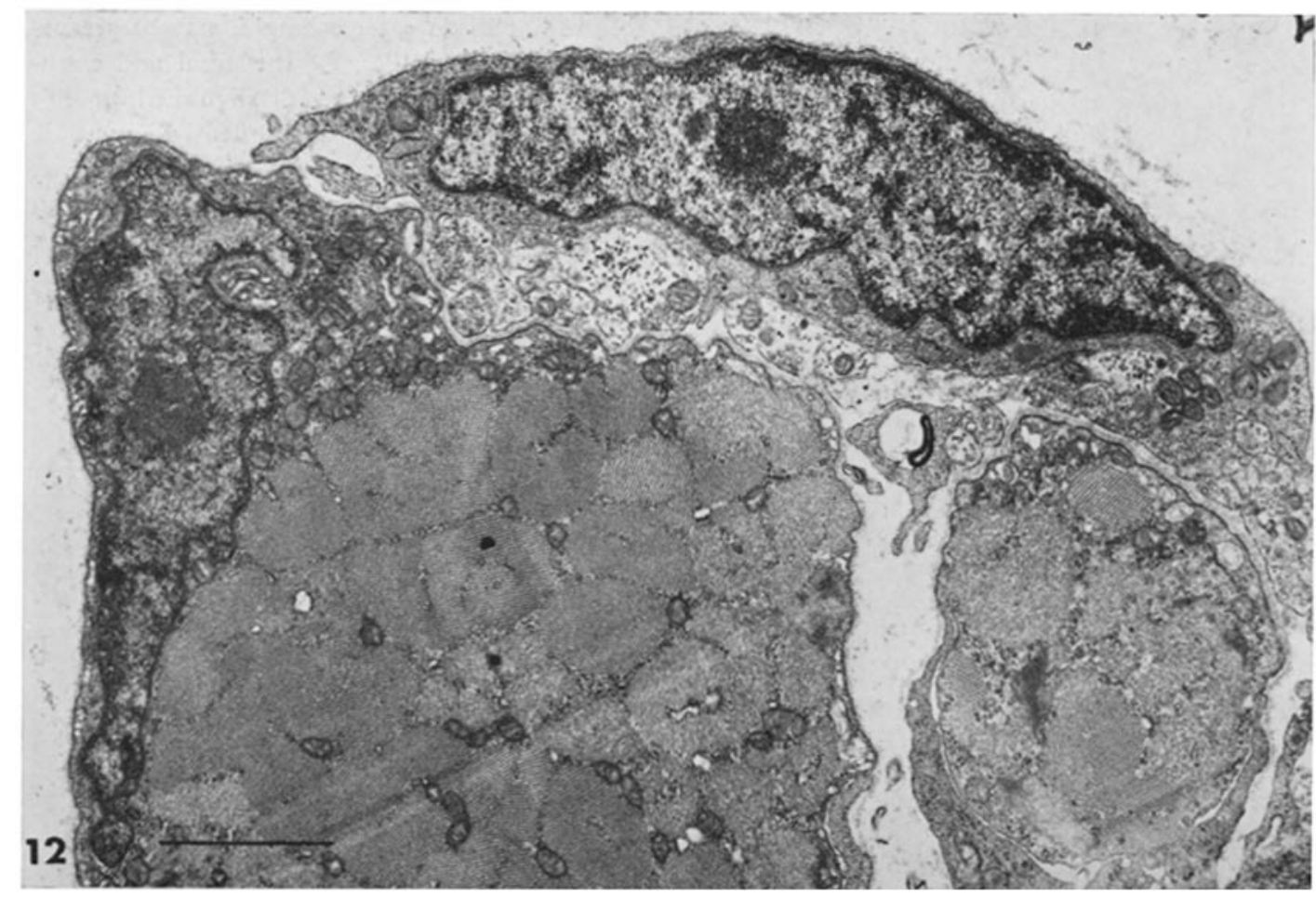

FIgURE 12 Birth. Numerous juxtaposed axon sprouts are contained within a single Schwann cell. They mutually innervate a large myofiber and an adjacent small myofiber. In the large myofiber a peripheral nucleus and some sarcoplasm lie to one side of the area of innervation. $\times 19,000$. Marker $=1 \mu$.

myofibers were observed with two nuclei in a single cross-section, one nucleus being adjacent to the endplate and the other nucleus being at the diametrically opposite side of the cell or disposed from the myofiber center to the opposite side as if migrating in that direction.

Intercellular spaces of up to $2 \mu$ separated small myofibers from neighboring large myofibers (Fig. 11). Terminal axon networks lying within single Schwann cells bridged these intercellular spaces and mutually innervated both large and small myofibers (Fig. 12), resembling the patterns of innervation of the groups of muscle cells at 18 and 20 days' gestation. The endplates on small myofibers was simpler in structure than those on large myofibers and were characterized by few primary synaptic clefts and scant accumulations of soleplate sarcoplasm. Secondary synaptic clefts, some of which were well developed, occurred along their postsynaptic membranes.

Groups of axon sprouts were traced in interrupted serial sections obtained from six separate blocks of muscle containing areas of innervation. In each case, all the muscle cells examined, regardless of size, had received nerves and most of these cells had evidence of early endplate differentiation.

At 10 days postpartum, there was abundant myelin within intramuscular nerves. At endplates of some large myofibers, terminal axon branches were separated from one another and lay within deep primary synaptic clefts (Fig. 14). Schwann cell processes covered nonsynaptic axon surfaces and contributed to the separation of axon sprouts from one another. Cell processes overlying Schwann cells were interpreted as perineural epithelium. Synaptic vesicles and osmiophilic, glycogen-like granules were abundant within the terminal axon branches. Endplate nuclei lay to one side of the postsynaptic membranes and were surrounded by soleplate sarcoplasm which was also distributed beneath neighboring synaptic clefts. The amount of sarcoplasm progressively diminished as the distance from the nuclei in- 


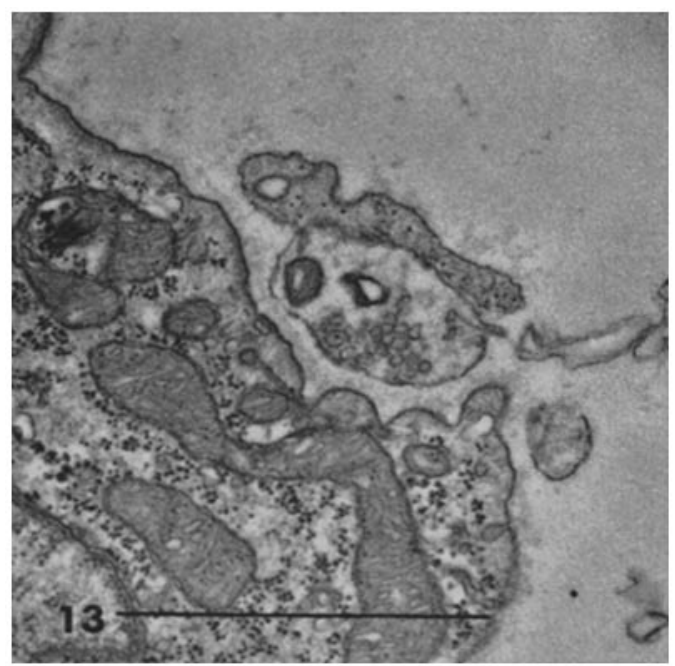

Figure 13 Birth. The postsynaptic membrane of a large myofiber forms a shallow primary synaptic cleft which partially surrounds an axon sprout. Short and wide secondary synaptic clefts arise from this primary cleft. $\times 38,000$. Marker $=1 \mu$.

creased. Elongated secondary synaptic clefts arose around many primary clefts at variable intervals. The developing motor endplates at this stage resembled mature endplates.

\section{DISCUSSION}

In a recent account of motor endplate formation, Teravainen (1968) observed that since the myoneural junction forms between two structurally different cells, it offers a unique opportunity to detect the morphogenetic events which lead to the formation of a synapse. Studies of synapse formation in the central nervous system initially appear to substantiate this remark for, although numerous electron-opaque junctions have been found between cell processes early in development, these junctions cannot be unequivocally correlated with the initiation of synapse formation because the cell processes bear no identifying features (3). The present study establishes that close membrane association and focal electron-opaque junctions form between nerve and muscle membranes early in development (Figs. 1-3). Correlation of these membrane specializations with initial events of motor endplate formation, however, is difficult and does not provide the unique opportunity suggested by Teravainen. Some of the close neuromuscular membrane associations observed at 16 days, for example, may be precursors of muscle spindles, for intrafusal and extrafusal fibers could not be distinguished at this stage. Furthermore, it was frequently impossible to decide whether the neuromuscular contact occurred between axon trunks or terminal axon sprouts as there was no way to identify the latter. That close membrane associations did occur between axon trunks and myotube membranes is illustrated in Fig. 5. This type of neuromuscular association appears to result from incomplete wrapping of nerves by Schwann cells and the absence of a basal lamina. With development of the Schwann sheath, early associations between the trunks of axons and myotube membranes cease to exist and they are, therefore, not directly related to the initiation of endplate formation. No close membrane associations were found between nerve and muscle at 18 days' gestation, at which time each group of muscle cells was surrounded by a continuous peripheral basal lamina. Between this stage of development and birth, new generations of muscle cells differentiated and formed motor endplates (Figs. 7 and 12). The plasma membranes of these small cells were, at 18 days, separated from immediate contact with nerve by a basal lamina. It seems very unlikely that the development of their motor endplates was initiated from the type of close neuromuscular association found at 16 days. Thus, although nerve and muscle membranes must initially be brought into stable relationship with each other for motor endplate differentiation to occur, the relationship of these preliminary connections to the ultimate formation of specific neuromuscular membrane differentiation is uncertain and requires further study.

Definitive evidence of endplate formation at 18 days' gestation was indicated by the presence of synaptic gaps with basal lamina between terminal axons and plasma membranes of large myotubes, by focal thickening and increased density of presumptive postsynaptic membranes (Fig. 8), and by histochemical evidence of acetylcholinesterase activity (Fig. 10). A single structure interpreted as a differentiating motor endplate was found at 16 days (Fig. 6) which resembled the early endplates described by Lentz (1968) and primitive rat motor endplates described by Teravainen (1968). Thus, Couteaux (1960) is incorrect when he suggests that motor endplate differentiation does not commence until after muscle cells mature 


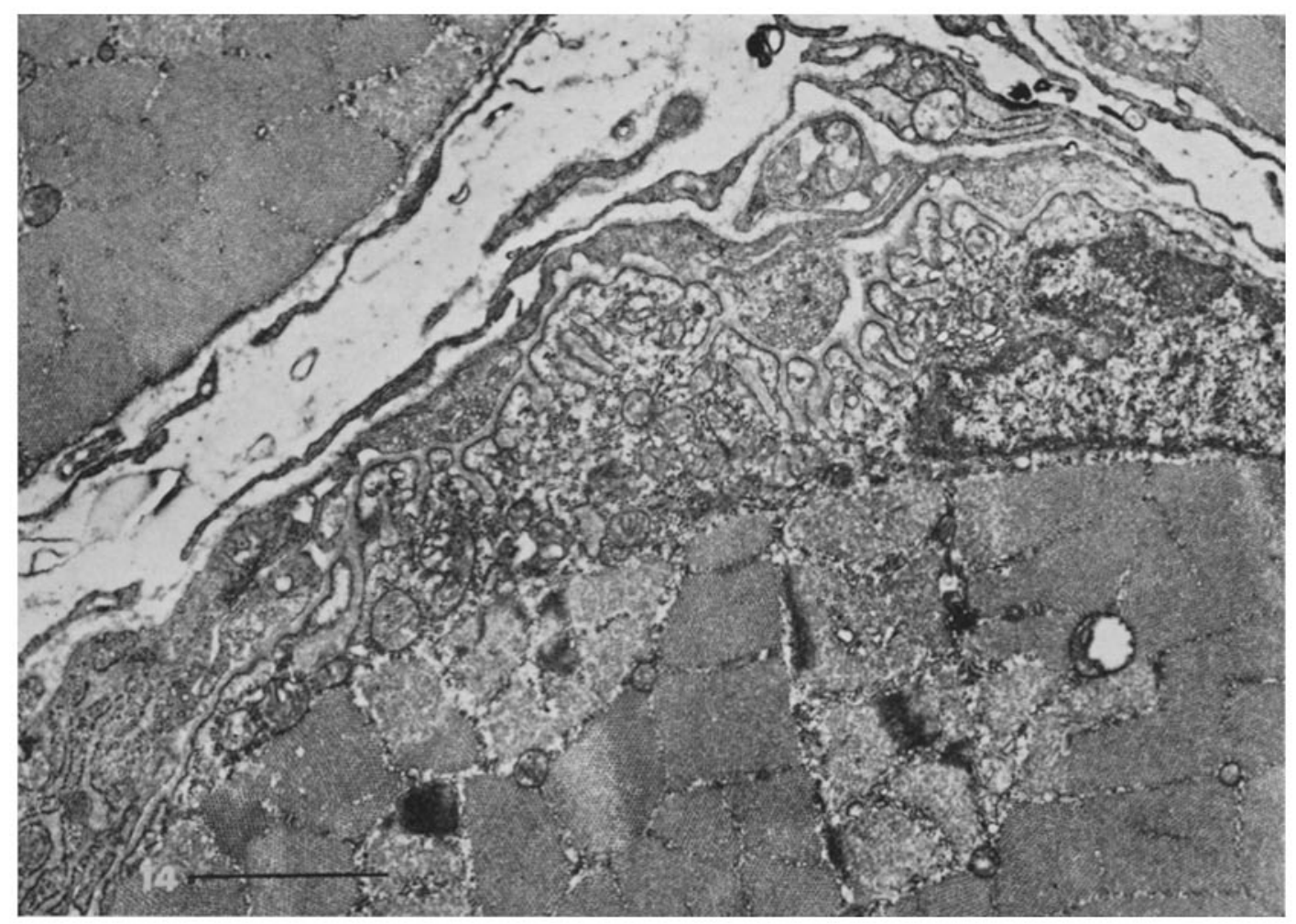

FrgURE 1410 days postpartum. This motor endplate resembles the mature synapse. Terminal axon branches are separated from each other by deep primary synaptic clefts. Schwann cell processes cover the nonsynaptic surfaces and further separate axon sprouts from one another. Soleplate sarcoplasm is distributed beneath the synaptic clefts. $\times 22,400$. Marker $=1 \mu$.

to myofibers. Thoracic movements of rat fetuses are first described after 18 days' gestation (10).

The progressive development of relationships between successive generations of developing muscle cells and associated nerve branches prompts speculation concerning the sites of differentiation of motor endplates on new generations of muscle cells. In our description of endplate formation at 18 days of gestation, primitive endplates were present on large myotubes, and associated small cells, interpreted as new generations of muscle cells, had axonal contacts but no definitive membrane thickening to indicate endplate formation. At birth, the new generations of small muscle cells had separated from the large muscle cells. The pattern of innervation, however, resembled that present at 18 days as neighboring large and small muscle cells received mutual innervation from groups of terminal axon sprouts contained within a single Schwann cell (Figs. 7 and 8), and definitive evidence of endplate formation was present on both large and small muscle cells. These observations suggest that the endplates of small myofibers develop from those areas of plasma membrane which border the postsynaptic membrane of large myotubes at 18 or 20 days' gestation. The evidence also leads to the conclusion that the site of formation of motor endplates on the new small muscle cells is determined by the local distribution of intercostal nerve and not by any specialization of the membrane of small myotubes to which the nerve grows.

Muscle cells which differentiate together within a group probably contract and relax in unison and do not begin functioning independently until after they have separated from each other (17). The present study raises the possibility that the activities of some neighboring muscle cells remain temporarily coordinated even after the muscle cells have separated. For example, at birth adja- 
cent large and small cells of this respiratory muscle which are mutually innervated by clusters of terminal axon sprouts contained within a single Schwann cell (Figs. 11 and 12) must function together. Myelination of peripheral nerve and the development of deep primary synaptic clefts surrounding individual axons, such as occurs at the endplates of some myofibers by 10 days postpartum (Fig. 13), effectively separates adjacent axon sprouts from one another. Until this developmental process is completed, specific neural control of individual myofibers is unlikely to be effective.

It would be interesting to know whether muscle cells which initially develop and function together within a group are subsequently related within the same motor unit. The pattern of developing innervation of several generations of muscle cells which initially are aggregated together and later separate suggests a possible orderly mechanism by which motor units may be assembled. Unfortunately, there is at present little evidence to support this hypothesis as electrophysiological data on the distribution of motor units within intercostal muscle are lacking and there is no way of knowing from the present study whether the clusters of axon sprouts which innervate neighboring large and small muscle cells in fetuses and neonates are derived from one or more neurons.

The origin of the aggregates of sarcoplasm and nuclei which form the soleplate has been discussed by a number of authors. On the basis of light microscope studies, Iwanaga (1925) and Csillik (1960, 1965) have suggested that Schwann cell and soleplate nuclei have a common origin. It is doubtful, however, that these authors could identify the various nuclei of cells associated with endplates as this region contains nuclei of Schwann cells, perineural cells, soleplate, and myofibers all within a small area. Consequently, we are unable to correlate their interpretations with those of the present study. By contrast, Couteaux (1960) and Wake (1964) considered that the aggregations of nuclei and sarcoplasm forming primitive soleplates resulted from local neural stimulation of muscle nuclei to undergo mitotic division. Their interpretation is untenable because most modern evidence indicates that multinucleation of striated muscle results from cell fusion, not nuclear division. The present study has shown that the presence of soleplate nuclei beside differentiating postsynaptic membranes coincides with the maturation of muscle cells to myofibers with peripheral nuclei. We obtained no morphological evidence to suggest that developing endplates possess a particular attraction for myotube nuclei. Many of the new generations of small muscle cells which clustered around the walls of large myotubes at 18 days' gestation lay adjacent to developing endplates. In a previous study (17), we proposed that these new generations of muscle cells may either separate and become independent cells or may fuse laterally into the substance of the large myotube and contribute to their multinucleation. Local fusion of several small cells in the vicinity of the primitive po tsynaptic membrane of a large myotube would produce an aggregation of sarcoplasm and nuclei comparable to a soleplate. The small cell with processes that enter invaginations of the large myotube in Fig. 8, for example, may be destined to fuse with the large myotube and contribute to the formation of a soleplate. In support of this hypothesis is the finding that this type of cellular relationship was not observed adjacent to the endplates on large myofibers at birth; instead, soleplate nuclei and sarcoplasm commonly formed a protuberance on the wall of the myofiber to one side of the postsynaptic membrane (Fig. 12) as if the result of lateral fusion.

This study was supported by the John A. Hartford Foundation Inc., New York, and the United States Public Health Service Training Grant 5T1-CA5097.

The authors wish to thank Miss S. Guyer for her competent assistance throughout the course of these investigations.

Received for publication 13 June 1968, and in revised form 17 February 1969.

\section{BIBLTOGR A PHY}

1. Barrnett, R. J., and G. E. Palade. 1959. Enzymatic activity in the $\mathrm{M}$ band. $J$. Biophys. Biochem. Cytol. 6:163.

2. Bleckschmidt, E., and Sh. Daikoku. 1966. Die

Entstehung der motorischen Innervation in der menschlichen Zungen Muskulatur. Acta Anat. 63:179.

3. Bunge, M. B., R. P. Bunge, and E. R. Peterson. 
1967. The onset of synapse formation in spinal cord cultures as studied by electron microscopy. Brain Res. 6:728.

4. Couteaux, R. 1960. In The Structure and Function of Muscle. G. H. Bourne, editor. Academic Press Inc., New York.

5. Csiller, B. 1960. Contribution to the development of the myoneural synapse. $Z$. Zellforsch. mikrosk. Anat. 52:150.

6. Csillik, B. 1965. Functional structure of the post-synaptic membrane in the myoneural junction. Publishing House of the Hungarian Academy of Sciences, Budapest.

7. Guajunco, J., 1942. Development of the human motor end plate. Contrib. Embryol. 30:129.

8. Dalton, A. J. 1955. A chrome-osmium fixative for electron microscopy. Anat. Rec. 121:281.

9. Dickson, L. M. 1940. Nerve endings in respiratory muscles of sheep. $J$. Anat. 74:268.

10. EAst, E. W. 1931. An anatomical study of the initiation of movement in rat embryos. Anat. Rec. 50:201.

11. Filagamo, G., and G. Gabella. 1967. The development of neuromuscular correlations in vertebrates. Arch. Biol. 78:9.

12. Hirano, H. 1967. Ultrastructural study on the morphogenesis of the neuromuscular junction in the skeletal muscle of the chick. $Z$. Zellforsch. mikrosk. Anat. 79:198.

13. Hirano, H., 1967. A histochemical study of the cholinesterase activity in the neuromuscular junction in developing chick skeletal muscle. Arch. Histol. japon. 28:89.

14. Iwanaga, I. 1925. Studien über die motorischen Nerven-endigungen. I. Ihre Histogenese. Mitt. Allgemaine Pathol. Pathologische Anat. 2: 257.

15. Karnovsky, M. J. 1965. A formaldehydegluteraldehyde fixative of high osmolality for use in electron microscopy. J. Cell Biol. 27: 137.

16. KELLY, A. M., and S. I. ZACKs. 1966. The development of the motor endplate in the rat. J. Cell Biol. 31A:114.

17. Kelly, A. M., and S. I. Zacks. 1969. The histogenesis of rat intercostal muscle. 42:135.
18. Kupfer, G., and G. B. Koelle. 1951. A histochemical study of cholinesterase during formation of the motor endplate of the albino rat. J. Exp. Zool. 116:397.

19. Lentz, T. L. 1968. Cytological and cytochemical studies of developing neuromuscular junction during limb regeneration of newt triturus. $J$. Cell Biol. 39:154a. (Abstr.)

20. Mummenthalar, M., and W. K. Engel. 1961. Cytological localization of cholinesterase in developing embryo skeletal muscle. Acta Anat. 47:274.

21. Pellegrino de Iraldi, A., and E. De Robertis. 1964. Ultrastructure and function of catecholamine containing systems. Excerpta Med. Int. Congr. Ser. No. 83. 355.

22. Peters, A., and A. R. Muir. 1959. The relationship between axons and Schwann cells during development of peripheral nerves in the rat. Quart. J. Exp. Physiol. 44:117.

23. Roth, F. T., and K. R. Porter. 1964. Yolk protein uptake in the oocyte of the mosquito Aedes Aegypti L. J. Cell Biol. 20:313.

24. Strauss, W. L., and G. Weddell. 1940. Nature of first visible contractions of the forelimb musculature in the rat foetus. $J$. Neurophysiol. $3: 358$.

25. Tello, J. F. 1922. Die Entstehung der motorischen und sensiblen Nervendigungen. $Z$. Gesamte Anat. 64:348.

26. Teravainen, H. 1968. Development of the myoneural junction in the rat. $Z$. Zellforsch. mikrosk. Anat. 87:249.

27. Trelstad, R., J. P. Revel, and E. Hay. 1967. Tight junctions between cells in the early chick embryo as visualized with the electron microscope. J. Cell Biol. 31:C6.

28. WAKE, K. 1964. Motor endplates in developing chick embryo skeletal muscle: Histological structure and histochemical localization of cholinesterase activity. Arch. Histol. jap. 25: 23.

29. Windle, W. F., W. L. Minear, M. F. Austin, and D. W. ORR. 1935. The origin and early development of somatic behaviour in the albino rat. Physiol-Zool. 8:156. 